\title{
Logarithmic correction to BMSFT entanglement entropy
}

\author{
Reza Fareghbal $^{1, \mathrm{a}}$, Pedram Karimi ${ }^{1, \mathrm{~b}}$ \\ ${ }^{1}$ Department of Physics, Shahid Beheshti University, G.C., Evin, Tehran 19839, Iran
}

Received: 22 February 2018 / Accepted: 23 March 2018 / Published online: 29 March 2018

(C) The Author(s) 2018

\begin{abstract}
Using Rindler method we derive the logarithmic correction to the entanglement entropy of a two dimensional BMS-invariant field theory (BMSFT). In particular, we present a general formula for extraction of the logarithmic corrections to both the thermal and the entanglement entropies. We also present a CFT formula related to the logarithmic correction of the BTZ inner horizon entropy which results in our formula after taking appropriate limit.
\end{abstract}

\section{Introduction}

One of the lessons of the AdS/CFT correspondence [1,2] is that the asymptotic symmetry of the asymptotically $\operatorname{AdS}$ spacetimes in $\mathrm{d}+1$ dimensions is the same as conformal symmetry in one dimension lower. One can use this idea to generalize the gauge/gravity duality beyond the AdS/CFT correspondence. Accordingly, in any non-AdS/non-CFT correspondence, the symmetry of the dual field theory should be the same as the asymptotic symmetry of the gravity solutions.

The asymptotic symmetry of asymptotically flat spacetimes were known long before Maldacena's conjecture. Such symmetries are known as BMS, which were first found at null infinity of four-dimensional asymptotically flat spacetimes [3-5], and later were generalized to the three-dimensional case [6]. Recently, Barnich and his collaborates [7-9] have shown that imposing just locally well-definiteness condition is enough to enhance both the translation and the rotation symmetry of the Poincare group at null infinity to an infinitedimensional symmetry group. In this work BMS group refers to this infinite-dimensional group which consists of superrotation and super-translation generators.

In one dimension lower than gravity theory, the BMS algebra is given by an ultra-relativistic contraction of the conformal algebra. Thus BMS symmetry can be the symmetry of a

\footnotetext{
a e-mail: r_fareghbal@sbu.ac.ir

b e-mail: pedramkarimie@gmail.com
}

lower dimensional field theory. It is proposed that the holographic dual of $\mathrm{d}+1$-dimensional asymptotically flat spacetimes are ultra-relativistic d-dimensional BMS-invariant field theories (BMSFTs) [10-12].

In flat-space holography, BMSFT entanglement entropy and its holographic dual were first studied in [13] and the entanglement entropy of a two-dimensional BMSFT was computed by a method similar to the CFT [14]. A holographic interpretation of the BMSFT entanglement entropy in [13] (similar to the Ryu-Takayanagi proposal in the context of AdS/CFT correspondence [15]) is given using ChernSimons formulation of three-dimensional flat-space gravity [16]. Other aspects of BMSFT entanglement entropy has been studied in $[17,18]$. A remarkable progress in this subject has been achieved recently in [19] where Rindler method [20] is used to derive not only the BMSFT entanglement entropy formula but also the holographic description in terms of some curves length in the bulk theory. The central idea of the Rindler method is to find local unitary transformations which map entangled states to the thermal states in the field theory. Then one can use the thermal entropy formula and find the entanglement entropy.

Calculation of thermal entropies in BMSFTs is performed using Cardy-like formula first introduced in [21]. Using Rindler method, the entanglement entropy is given by Cardylike formula. Similar to the Cardy-formula in CFT, the saddle point approximation is used to derive this formula. Thus, it is possible to improve approximation and find possible corrections of this formula. In [22], employing a method first used for the Cardy formula in [23] $]^{1}$, logarithmic correction to the Cardy-like formula has been derived.

\footnotetext{
1 We note however that the Cardy (high temperature) limit is not always reliable for extracting the black hole entropy [24]. On the other hand, in two dimensional CFTs the logarithmic corrections are only universal in the Cardy regime. From the dual three dimensional gravity perspective, the universality of the correction is usually because heat kernels do not contain logarithmic terms [25]. Thus the logarithmic correction to the BMSFT thermal and entanglement entropies are universal in the limit that BMSFT Cardy-like formula is reliable.
} 
In this paper, we use the logarithmic correction to Cardylike formula along with Rindler method to find the logarithmic correction of BMSFT entanglement entropy. Similar to the leading term, this correction depends on central charges of BMS algebra, the interval of the sub-system and the cutoff. However, the interesting point is that we can rewrite the corrections in a universal form:

$S=S_{0}-3 \log \left(C_{M}^{\frac{1}{3}} \frac{\partial S_{0}}{\partial C_{L}}\right)$

where $S_{0}$ is the leading term and $C_{L}$ and $C_{M}$ are the central charges of BMS algebra. $S$ can be both of the thermal entropy (which is given by the Cardy-like formula) and the entanglement entropy. This formula works for all BMSFTs on plane or cylinder at zero or finite temperature.

The entanglement entropy of the boundary theory can be used to reconstruct the bulk dynamics [26,27]. In this view, the logarithmic correction of the entanglement entropy might be helpful to find quantum correction to the Einstein field equations. This paper is the first step on this road. The idea is to use the first law of the entanglement entropy as the analogue of the first law of (black hole) thermodynamics [2628]. The better understanding of BMSFT modular hamiltonian might allow us to achieve not only the classical bulk dynamics but also the quantum correction to the Einstein equation without the cosmological constant.

One approach to study Flat/BMSFT is to take limit from the AdS/CFT calculations. According to the proposal of [12], the flat space limit in the gravity side corresponds to taking an ultra-relativistic limit from the CFT calculations. Hence one can find all BMSFT formulas by taking limit from a CFT counterpart. It was shown in $[29,30]$ that the Cardy-like formula of BMSFT is given by taking limit from a formula in the CFT which is related to the inner horizon entropy of the gravity solution. If we assume the same relation for the logarithmic correction, the logarithmic term in (1.1) is related to a logarithmic correction in the entropy of the inner horizon. We propose a suitable logarithmic correction in the inner horizon formula which corresponds to the logarithmic term of (1.1) after taking limit. Using logarithmically corrected inner and outer horizon entropy formulas we can calculate their multiplication. The observation is that the previously known result, the multiplication being mass independent for the Einstein gravity [31] (see also [32]), is violated in the presence of the logarithmic terms.

The structure of this paper is as follows: In section two we review the results of [19] which uses the Rindler method to derive the BMSFT entanglement entropy and its holographic description. In section three we review the derivation of BMSFT Cardy-like formula and its logarithmic correction. In section four we put together the results of section two and three to derive the logarithmic correction of BMSFT entanglement entropy. Section five is devoted to the deriva- tion of the BMSFT entanglement entropy formula by taking flat-space limit.

\section{Entanglement entropy of BMSFT using rindler method}

One of the advantages of the Rindler method is the convenience it provides for the calculation of entanglement entropy in the context of gauge/gravity duality. In this view, the thermal entropy of the boundary theory is mapped to the horizon entropy of the black hole (object) in the gravity side. Thus, one can use it to prove Ryu-Takayanagi (RT) formula for the holographic entanglement of CFTs. Moreover, since many thermal properties of the gravitational systems are known for the non-AdS cases, we expect that the Rindler method gives a lot of insights to find the analogue of RT formula for the dualities beyond the AdS/CFT correspondence.

In Rindler method, the asymptotic symmetries play an essential role. We are interested in BMSFTs which are proposed to be the holographic dual of asymptotically flat spacetimes. In our case, a Rindler transformation is of the form of BMS transformation and final coordinates are invariant under specific thermal identifications. These transformations act like unitary operators $U_{\mathcal{R}}$ on the fields and map the reduced density matrix in the subregion $\mathcal{A}$ (which we want to calculate the entanglement entropy for) to a thermal density matrix in the interval $\mathcal{B}$,

$\rho_{\mathcal{A}}=U_{\mathcal{R}} \rho_{\mathcal{B}} U_{\mathcal{R}}^{-1}$.

Since the unitary transformations do not change the entropy, the thermal entropy of the subregion $\mathcal{B}$ is the same as entanglement entropy of the subregion $\mathcal{A}$.

A two-dimensional BMSFT has the following symmetry [33]:

$\tilde{u}=\partial_{\phi} f(\phi) u+g(\phi)$,

$\tilde{\phi}=f(\phi)$,

where $f(\phi)$ and $g(\phi)$ are arbitrary functions of the original coordinate $(u, \phi)$. The BMS algebra is given by using infinitesimal BMS transformation as

$$
\begin{aligned}
{\left[L_{n}, L_{m}\right] } & =(n-m) L_{n+m}+\frac{C_{L}}{12} n\left(n^{2}-1\right) \delta_{n+m, 0}, \\
{\left[L_{n}, M_{m}\right] } & =(n-m) M_{n+m}+\frac{C_{M}}{12} n\left(n^{2}-1\right) \delta_{n+m, 0}, \\
{\left[M_{n}, M_{m}\right] } & =0 .
\end{aligned}
$$

where for a BMSFT on a plane with coordinates $(u, \phi)$ the generators $L_{n}$ and $M_{n}$ are given by 


$$
\begin{aligned}
L_{n} & =-u(n+1) \phi^{n} \partial_{u}-\phi^{n+1} \partial_{\phi} \\
M_{n} & =\phi^{n+1} \partial_{u} .
\end{aligned}
$$

The global part of this algebra is identified with $n=0, \pm 1$.

A Rindler transformation is of the form $\tilde{x}=T(x)$, but it should be invariant under some imaginary identification (thermal identification) of the new coordinate $\tilde{x}^{i} \sim \tilde{x}^{i}+$ $i \tilde{\beta}^{i}$. Moreover, vectors $\partial_{\tilde{x}^{i}}$ annihilate the vacuum and hence should be written as the linear combination of the global part of the BMS algebra:

$\partial_{\tilde{x}^{i}}=\sum_{n=-1}^{1}\left(b_{n} L_{n}+d_{n} M_{n}\right)$.

Using (2.2) and (2.5) we conclude that

$\partial_{\phi} f(\phi)=\frac{1}{Y}, \quad \partial_{\phi} g(\phi)=-\frac{T}{Y^{2}}$,

where

$$
\begin{aligned}
& Y=-b_{-1}-b_{0} \phi-b_{1} \phi^{2}, \\
& T=d_{-1}+d_{0} \phi+d_{1} \phi^{2} .
\end{aligned}
$$

Solutions to (2.6) determine vector $\partial_{\tilde{x}^{i}}$. It is assumed that entangled region is given by $\mathcal{A}=\left\{\left(\frac{-l_{\phi}}{2}, \frac{-l_{u}}{2}\right) \cup\left(\frac{l_{\phi}}{2}, \frac{l_{u}}{2}\right)\right\}$. We can use the following constraints to find vector $\partial_{\tilde{x}^{i}}$ and the geometric (modular) flow $k_{t}=-\tilde{\beta_{\phi}} \partial_{\tilde{\phi}}+\tilde{\beta_{u}} \partial_{\tilde{u}}$ :

1. The finite interval on $\mathcal{A}$ should be mapped to the infinite interval on $\mathcal{B}$,

$$
\begin{gathered}
\left(\frac{-l_{\phi}}{2}, \frac{-l_{u}}{2}\right) \rightarrow(-\infty,-\infty), \\
\left(\frac{l_{\phi}}{2}, \frac{l_{u}}{2}\right) \rightarrow(\infty, \infty) .
\end{gathered}
$$

2. The origin of the entangled interval is mapped to the origin of the thermal interval

$$
(0,0) \rightarrow(0,0)
$$

3. The thermal interval (tilde coordinate) obeys a thermal identification of the following form

$$
(\tilde{\phi}, \tilde{u}) \sim\left(\tilde{\phi}+i \tilde{\beta}_{\phi}, \tilde{u}-i \tilde{\beta}_{u}\right)
$$

4. The modular flow $k_{t}$ vanishes on the boundary of the entangled region

$k_{t}(\partial \mathcal{A})=0 \Rightarrow\left\{\begin{array}{l}k_{t}\left(\frac{-l_{\phi}}{2}, \frac{-l_{u}}{2}\right)=0 \\ k_{t}\left(\frac{l_{\phi}}{2}, \frac{l_{u}}{2}\right)=0\end{array}\right.$
Using these conditions, [19] completely determines the Rindler transformation and modular flow of a BMSFT on the plane as below:

$$
\begin{aligned}
\tilde{\phi}= & \frac{\tilde{\beta}_{\phi}}{\pi} \tanh ^{-1} \frac{2 \phi}{l_{\phi}}, \\
\tilde{u}+\frac{\tilde{\beta}_{u}}{\tilde{\beta}_{\phi}} \tilde{\phi}= & \frac{2 \tilde{\beta}_{\phi}\left(u l_{\phi}-l_{u} \phi\right)}{\pi\left(l_{\phi}^{2}-4 \phi^{2}\right)}, \\
k_{t}= & -\tilde{\beta}_{\phi} \partial_{\phi}+\tilde{\beta}_{u} \partial_{u} \\
= & \frac{-\pi}{2 l_{\phi}}\left(\left(l_{\phi}^{2}-4 \phi^{2}\right) \partial_{\phi}\right. \\
& \left.+\left(l_{u} l_{\phi}+4 \frac{l u}{l_{\phi}} \phi^{2}-8 u \phi\right) \partial_{u}\right)
\end{aligned}
$$

For a BMSFT with identification of coordinates as

$(\tilde{u}, \tilde{\phi}) \sim(\tilde{u}+i \bar{a}, \tilde{\phi}-i a) \sim(\tilde{u}+2 \pi \bar{b}, \tilde{\phi}-2 \pi b)$,

The degeneracy of states is given by a Cardy-like formula $[19,21,34]$ (see next section).

$S_{\bar{b} \mid b}(\bar{a} \mid a)=\frac{-\pi^{2}}{3}\left(C_{L} \frac{b}{a}+C_{M} \frac{(\bar{a} b-a \bar{b})}{a^{2}}\right)$.

Using (2.18), the entanglement entropy of a BMSFT on the plane for the interval $\mathcal{A}=\left\{\left(\frac{-l_{\phi}}{2}+\epsilon_{\phi}, \frac{-l_{u}}{2}+\epsilon_{u}\right) \cup\left(\frac{l_{\phi}}{2}-\right.\right.$ $\left.\left.\epsilon_{\phi}, \frac{-l_{u}}{2}-\epsilon_{\phi}\right)\right\}$ becomes [19]

$S_{E E}=\frac{C_{L}}{6} \log \frac{l_{\phi}}{\epsilon_{\phi}}+\frac{C_{M}}{6}\left(\frac{l_{u}}{l_{\phi}}-\frac{\epsilon_{u}}{\epsilon_{\phi}}\right)$,

where $\epsilon_{u}$ and $\epsilon_{\phi}$ are ultraviolet cut-offs in $u$ and $\phi$ coordinates. Similarly the entanglement entropy for finite temperature BMSFT on the cylinder has been calculated in [19].

\section{Logarithmic correction to Cardy-like formula of BMSFT}

The entropy of CFT thermal states is calculated using Cardy formula. Using the saddle point approximation, it is possible to find a similar formula for the degeneracy of thermal states in a BMSFT [19,21,34]. Due to the Rindler method, any correction to Cardy-like formula has influence on the entanglement entropy formula. In this section, we review the logarithmic correction to Cardy-like formula [22] and then use it to find the logarithmic correction to the entanglement entropy. Method of [22] is based on [23] that introduces the first order logarithmic correction to the Cardy formula (For a calculation of all order corrections see [35]) 
We start from the modular invariant partition function of BMSFT on a torus defined by

$$
\begin{aligned}
Z_{0}\left(\hat{\beta}_{u} \mid \hat{\beta}_{\phi}\right) & =\operatorname{Tr} e^{-\hat{\beta}_{u}\left(M_{0}-\frac{C_{M}}{24}\right)+\hat{\beta}_{\phi}\left(L_{0}-\frac{C_{L}}{24}\right)} \\
& =e^{\hat{\beta}_{u} \frac{C_{M}}{24}-\hat{\beta}_{\phi} \frac{C_{L}}{24}} Z\left(\hat{\beta}_{u} \mid \hat{\beta}_{\phi}\right),
\end{aligned}
$$

where $Z\left(\hat{\beta}_{u} \mid \hat{\beta}_{\phi}\right)$ is

$$
\begin{aligned}
Z\left(\hat{\beta}_{u} \mid \hat{\beta}_{\phi}\right) & =\operatorname{Tr} e^{-\hat{\beta}_{u} M_{0}+\hat{\beta}_{\phi} L_{0}} \\
& =\sum_{h_{M}, h_{L}} e^{\left(-\hat{\beta}_{u} h_{M}+\hat{\beta}_{\phi} h_{L}\right)} d\left(h_{M}, h_{L}\right),
\end{aligned}
$$

and identification of torus are

$$
(\hat{u}, \hat{\phi}) \sim\left(\hat{u}+i \hat{\beta}_{u}, \hat{\phi}-i \hat{\beta}_{\phi}\right) \sim(\hat{u}, \hat{\phi}-2 \pi) .
$$

Here, $h_{L}$ and $h_{M}$ are respectively the eigenvalues of $L_{0}$ and $M_{0}$. It is shown that the BMS modular invariant partition function satisfies [19]

$Z_{0}\left(\hat{\beta}_{u} \mid \hat{\beta}_{\phi}\right)=Z_{0}\left(-4 \pi^{2} \frac{\hat{\beta}_{u}}{\hat{\beta}_{\phi}^{2}} \mid \frac{4 \pi^{2}}{\hat{\beta}_{\phi}}\right)$.

Plugging equation (3.4) into (3.1) gives,

$$
\begin{aligned}
Z\left(\hat{\beta}_{u} \mid \hat{\beta}_{\phi}\right)= & e^{\hat{\beta}_{u} \frac{C_{M}}{24}-\hat{\beta}_{\phi} \frac{C_{L}}{24}-\frac{\pi^{2} \hat{\beta}_{u} C_{M}}{6 \hat{\beta}_{\phi}^{2}}-\frac{\pi^{2} C_{L}}{6 \hat{\beta}_{\phi}} Z} \\
& \times\left(-4 \pi^{2} \frac{\hat{\beta}_{u}}{\hat{\beta}_{\phi}^{2}} \mid \frac{4 \pi^{2}}{\hat{\beta}_{\phi}}\right) .
\end{aligned}
$$

By using the inverse Laplace transformation in last term of (3.2) we find

$$
\begin{aligned}
d\left(h_{L}, h_{M}\right)= & \int d \hat{\beta}_{u} d \hat{\beta}_{\phi} e^{\hat{\beta}_{u} c_{M}-\hat{\beta}_{\phi} \frac{C_{L}}{24}-\frac{\pi^{2} \hat{\beta}_{u} C_{M}}{6 \hat{\beta}_{\phi}^{2}}-\frac{\pi^{2} C_{L}}{6 \hat{\beta}_{\phi}}+\hat{\beta}_{u} h_{M}-\hat{\beta}_{\phi} h_{L}} Z \\
& \times\left(-4 \pi^{2} \frac{\hat{\beta}_{u}}{\hat{\beta}_{\phi}^{2}} \mid \frac{4 \pi^{2}}{\hat{\beta}_{\phi}}\right) .
\end{aligned}
$$

In order to simplify (3.6), we use two approximations. First, we consider large charges which yields

$$
\begin{aligned}
d\left(h_{L}, h_{M}\right)= & \int d \hat{\beta}_{u} d \hat{\beta}_{\phi} e^{-\frac{\pi^{2} \hat{\beta}_{u} C_{M}}{6 \hat{\beta}_{\phi}^{2}}-\frac{\pi^{2} C_{L}}{6 \hat{\beta}_{\phi}}+\hat{\beta}_{u} h_{M}-\hat{\beta}_{\phi} h_{L}} Z \\
& \times\left(-4 \pi^{2} \frac{\hat{\beta}_{u}}{\hat{\beta}_{\phi}^{2}} \mid \frac{4 \pi^{2}}{\hat{\beta}_{\phi}}\right) .
\end{aligned}
$$

Then, we approximate (3.6) around the saddle point given by

$$
\left(\hat{\beta}_{\phi}^{s}\right)^{2}=\frac{\pi^{2} C_{M}}{6 h_{M}}, \quad \hat{\beta}_{u}^{s}=\frac{\hat{\beta}_{\phi}^{s}}{2 h_{M} C_{M}}\left(C_{M} h_{L}-C_{L} h_{M}\right) .
$$

Finally, the entropy or Cardy like formula for BMSFT reads as $^{2}$

$S^{0}=\log d\left(h_{L}, h_{M}\right)=-\frac{\pi^{2}}{3\left(\hat{\beta}_{\phi}^{s}\right)^{2}}\left(C_{M} \hat{\beta}_{u}^{s}+C_{L} \hat{\beta}_{\phi}^{s}\right)$.

To find logarithmic correction we expand the integral (3.7) around the saddle point (3.8) up to quadratic term:

$d\left(h_{L}, h_{M}\right)=e^{S^{0}} \int d \hat{\beta}_{u} d \hat{\beta}_{\phi} e^{\frac{1}{2}\left(X^{2}-Y^{2}\right)}$,

where

$X=\frac{\pi C_{M}}{3 \hat{\beta}_{\phi}^{s} A}\left(\hat{\beta}_{u}-\hat{\beta}_{u}^{s}\right)$,

$Y=\frac{\pi A}{\left(\hat{\beta}_{\phi}^{s}\right)^{2}}\left[-\left(\hat{\beta}_{\phi}-\hat{\beta}_{\phi}^{s}\right)+\frac{C_{M} \hat{\beta}_{\phi}^{s}}{3 A^{2}}\left(\hat{\beta}_{u}-\hat{\beta}_{u}^{s}\right)\right]$,

$A=\sqrt{C_{M} \hat{\beta}_{u}^{s}+\frac{1}{3} C_{L} \hat{\beta}_{\phi}^{s}}$.

Using (3.11), Eq. (3.10) takes the form

$d\left(h_{L}, h_{M}\right)=e^{S^{0}}\left(-\frac{\pi^{2} C_{M}}{3\left(\hat{\beta}_{\phi}^{s}\right)^{3}}\right)^{-1} \int d X d Y e^{\frac{X^{2}-Y^{2}}{2}}$.

In the above equation the result of integration is just a number. Thus we find correction to the entropy up to a constant as [22]

$$
\begin{aligned}
S= & \log d\left(h_{L}, h_{M}\right) \\
= & -\frac{\pi^{2}}{3\left(\hat{\beta}_{\phi}^{s}\right)^{2}}\left(C_{M} \hat{\beta}_{u}^{s}+C_{L} \hat{\beta}_{\phi}^{s}\right) \\
& -3 \log \left(-\frac{C_{M}^{\frac{1}{3}}}{\hat{\beta}_{\phi}^{s}}\right)+\text { constant. }
\end{aligned}
$$

The interesting point is that the logarithmically corrected term can be rewritten (up to a constant) as the derivative of the leading term with respect to $C_{L}$ :

$S=S_{0}-3 \log \left(C_{M}^{\frac{1}{3}} \frac{\partial S_{0}}{\partial C_{L}}\right)$.

\section{Logarithmic correction to entanglement entropy}

In this section, we put together the results from sections two and three to find the logarithmic correction of BMSFT entanglement entropy. As mentioned before, the idea is to map entangled states to thermal states and then calculate entropy. The entropy is computed using BMSFT Cardy-like formula (3.14) which also has a logarithmic correction. In the derivation of (3.14) the identification of coordinates (3.3) plays

${ }^{2}$ It is assumed that the partition function is slowly varying at the extremum. 
an essential role. It is possible to use (3.14) for finding the degeneracy of thermal states with more generic identification of coordinates as

$$
(\tilde{u}, \tilde{\phi}) \sim(\tilde{u}+i \bar{a}, \tilde{\phi}-i a) \sim(\tilde{u}+2 \pi \bar{b}, \tilde{\phi}-2 \pi b)
$$

The coordinate change between $(\tilde{u}, \tilde{\phi})$ and $(\hat{u}, \hat{\phi})$ is a BMS transformation,

$\hat{\phi}=\frac{\tilde{\phi}}{b}, \quad \hat{u}=\frac{\tilde{u}}{b}+\frac{\bar{b}}{b^{2}} \tilde{\phi}$,

where

$\hat{\beta}_{\phi}=\frac{a}{b}, \quad \hat{\beta}_{u}=\frac{\bar{a} b-a \bar{b}}{b^{2}}$.

Thus Cardy-like formula (3.14) can be written as

$S=-\frac{\pi^{2}}{3}\left(C_{L} \frac{b}{a}+C_{M} \frac{\bar{a} b-a \bar{b}}{a^{2}}\right)-3 \log \left(-C_{M}^{\frac{1}{3}} \frac{b}{a}\right)$

In order to find the logarithmic correction of BMSFT entanglement entropy, it is enough to map the entanglement entropy to a thermal entropy and then use (4.4). As it was reviewed in Sect. 2, the Rindler transformation which governs this map is determined in such a way that finally induces the thermal identification (2.12). Comparing (2.12) to (4.1) shows that

$a=\tilde{\beta}_{\phi}, \quad \bar{a}=\tilde{\beta}_{u}$.

The values of $\tilde{\beta}_{\phi}, \tilde{\beta}_{u}$ and $b, \bar{b}$ depend on the details of the Rindler transformation. These are given in terms of the cutoffs and the interval for which entanglement entropy is calculated.

Starting from a regulated interval in the BMSFT given by

$$
\left(-\frac{l_{u}}{2}+\epsilon_{u},-\frac{l_{\phi}}{2}+\epsilon_{\phi}\right) \rightarrow\left(\frac{l_{u}}{2}-\epsilon_{u}, \frac{l_{\phi}}{2}-\epsilon_{\phi}\right),
$$

the Rindler transformation yields the following results [19]:

- For the zero temperature BMSFT on the plane we have

$$
\begin{aligned}
& a=\tilde{\beta}_{\phi}=-\frac{2 \pi^{2}}{\log \frac{l_{\phi}}{\epsilon_{\phi}}}, \quad \bar{a}=\tilde{\beta}_{u}=-\frac{\tilde{\beta}_{\phi}^{2}}{2 \pi^{2}}\left(\frac{l_{u}}{l_{\phi}}-\frac{\epsilon_{u}}{\epsilon_{\phi}}\right), \\
& b=-\frac{\tilde{\beta}_{\phi}}{2 \pi^{2}} \log \frac{l_{\phi}}{\epsilon_{\phi}}, \\
& \bar{b}=\frac{1}{2 \pi^{2}}\left(\frac{\tilde{\beta}_{\phi} l_{u}}{l_{\phi}}-\frac{\tilde{\beta}_{\phi} \epsilon_{u}}{\epsilon_{\phi}}-\tilde{\beta}_{u} \log \frac{l_{\phi}}{\epsilon_{\phi}}\right)
\end{aligned}
$$

Then the logarithmically corrected Cardy-like formula (4.4) becomes

$$
\begin{aligned}
S_{E E}= & \frac{C_{L}}{6} \log \frac{l_{\phi}}{\epsilon_{\phi}}+\frac{C_{M}}{6}\left(\frac{l_{u}}{l_{\phi}}-\frac{\epsilon_{u}}{\epsilon_{\phi}}\right) \\
& -3 \log \left(C_{M}^{\frac{1}{3}} \log \frac{l_{\phi}}{\epsilon_{\phi}}\right)+\text { constant. }
\end{aligned}
$$

The third term in the above formula is the calculated correction.

- For the finite temperature BMSFT with identification

$$
(u, \phi) \sim\left(u+i \beta_{u}, \phi-i \beta_{\phi}\right),
$$

we can use the results of [19] and Eq. (4.4) to write

$$
\begin{aligned}
S_{E E}= & \frac{C_{L}}{6} \log \left(\frac{\beta_{\phi}}{\pi \epsilon_{\phi}} \sinh \frac{\pi l_{\phi}}{\beta_{\phi}}\right) \\
& +\frac{C_{M}}{6} \frac{1}{\beta_{\phi}}\left[\pi\left(l_{u}+\frac{\beta_{u}}{\beta_{\phi}} l_{\phi}\right) \operatorname{coth} \frac{\pi l_{\phi}}{\beta_{\phi}}-\beta_{u}\right] \\
& -\frac{C_{M} \epsilon_{u}}{6 \epsilon_{\phi}} \\
& -3 \log \left(C_{M}^{\frac{1}{3}} \log \left(\frac{\beta_{\phi}}{\pi \epsilon_{\phi}} \sinh \frac{\pi l_{\phi}}{\beta_{\phi}}\right)\right)+\text { constant. }
\end{aligned}
$$

The forth term in the above equation is the obtained correction for finite temperature.

- For the zero temperature BMSFT on the cylinder, the entanglement entropy with logarithmic correction reads as

$$
\begin{aligned}
S_{E E}= & \frac{C_{L}}{6} \log \left(\frac{2}{\epsilon_{\phi}} \sin \frac{l_{\phi}}{2}\right)+\frac{C_{M}}{12}\left(l_{u} \cot \frac{l_{\phi}}{2}-\frac{2 \epsilon_{u}}{\epsilon_{\phi}}\right) \\
& -3 \log \left(C_{M}^{\frac{1}{3}} \log \left(\frac{2}{\epsilon_{\phi}} \sin \frac{l_{\phi}}{2}\right)\right)+\text { costant. }
\end{aligned}
$$

It is clear from all the above cases that up to a constant, the entanglement entropy formula including the logarithmic correction is given by

$$
S_{E E}=S_{0}-3 \log \left(C_{M}^{\frac{1}{3}} \frac{\partial S_{0}}{\partial C_{L}}\right)
$$

This formula is also valid for the thermal entropy when $S_{0}$ is the Cardy-like formula. Thus, we propose a universal form for the logarithmic correction of entanglement entropy and thermal entropy. We expect the same common form for the CFT case. In the next section, we propose a similar form of logarithmic correction to CFT thermal and entanglement entropy and try to find (4.13) by taking limit from CFT formula. 


\section{Logarithmic correction of BMSFT entropy by taking limit from CFT counterpart}

For a two dimensional CFT with central charges $c$ and $\bar{c}$ and right and left temperatures $\beta$ and $\bar{\beta}$, the Cardy formula is

$S_{0}=\frac{\pi^{2}}{3}\left(\frac{c}{\beta}+\frac{\bar{c}}{\bar{\beta}}\right)$.

Using AdS/CFT correspondence, this formula results in the same entropy as the outer horizon entropy of asymptotically AdS black holes in the gravity side. The logarithmic correction to (5.1) was evaluated in [23]:

$S_{\text {log }}=-\frac{3}{2} \log \left(\frac{c^{1 / 3}}{\beta}\right)-\frac{3}{2} \log \left(\frac{\bar{c}^{1 / 3}}{\bar{\beta}}\right)+$ constant.

Employing (5.1) and (5.2) we can write

$$
\begin{aligned}
S=S_{0}+S_{l o g}= & S_{0}-\frac{3}{2} \log \left(c^{1 / 3} \frac{\partial S_{0}}{\partial c}\right) \\
& -\frac{3}{2} \log \left(\bar{c}^{1 / 3} \frac{\partial S_{0}}{\partial \bar{c}}\right)+\text { constant. }
\end{aligned}
$$

Using Rindler method we can expect the same formula for the logarithmically corrected CFT entanglement entropy. Thus we propose (5.3) as a universal formula that can be used for both the thermal entropy and the entanglement entropy.

It is known that taking flat space limit from the asymptotically AdS spacetimes (written in the appropriate coordinate) yields asymptotically flat spacetimes. It is proposed in [12] that the flat space limit in the bulk corresponds to taking the ultra-relativistic limit in the boundary CFT. In other words, BMSFT is given by taking ultra-relativistic limit from the CFT. Starting from conformal algebra in two dimensions, one can introduce an ultra-relativistic contraction and generate BMS algebra [12]. The relation between central charges of conformal algebra and BMS algebra is given by

$C_{L}=\lim _{\epsilon \rightarrow 0}(c-\bar{c}), \quad C_{M}=\lim _{\epsilon \rightarrow 0} \epsilon(c+\bar{c})$

where $\epsilon$ is a dimensionless parameter which corresponds to $G / \ell$ on the gravity side. $G$ is the Newton constant and $\ell$ is the AdS-radius. Thus $\epsilon \rightarrow 0$ in the boundary corresponds to $\ell \rightarrow \infty$.

It is plausible to find all BMSFT quantities by taking limit from the CFT counterparts. Thus one can look for the possible relation between (4.13) and (5.3). However, assuming $S_{0}$ in (5.3) as the Cardy formula (5.1) and using (5.4) does not result in the Cardy-like formula (3.9). It was shown in [29] and [30] that the appropriate formula which its limit yields the Cardy-like formula is

$$
S_{\text {inner }}^{0}=\frac{\pi^{2}}{3}\left(\frac{c}{\beta}-\frac{\bar{c}}{\bar{\beta}}\right) .
$$

For taking limit we should scale the temperatures as below:

$$
\beta_{u}=\lim _{\epsilon \rightarrow 0}\left(\frac{\beta-\bar{\beta}}{2 \epsilon}\right), \quad \beta_{\phi}=\lim _{\epsilon \rightarrow 0}-\left(\frac{\beta+\bar{\beta}}{2}\right) .
$$

Using AdS/CFT correspondence, (5.5) corresponds to the inner horizon entropy of asymptotically AdS black holes. Thus, we expect that taking limit from (5.3) which is written for the outer horizon does not yield (4.13). In the rest of this section, we introduce appropriate formula which taking limit from it result in (4.13).

Let us start from $S^{0}$ in (4.13). When it is the thermal entropy given by the Cardy-like formula, the corresponding formula in the CFT will be (5.5). The question is then what is the corresponding CFT formula when $S^{0}$ in (4.13) is not the leading order of entanglement entropy?

It is known that taking ultra-relativistic limit from the entanglement entropy of CFT is not well-defined. For example, for a zero temperature CFT on a plane, the entanglement entropy of an interval

$-\frac{R}{2}(\cosh \kappa, \sinh \kappa) \rightarrow \frac{R}{2}(\cosh \kappa, \sinh \kappa)$,

is given by [36]

$S_{E E}=\frac{c+\bar{c}}{2} \log \frac{R}{\epsilon_{R}}-\frac{c-\bar{c}}{6} \kappa$

where $\epsilon_{R}$ is a cut-off. Using (5.4) together with ultrarelativistic contraction $t \rightarrow \epsilon t$ shows that (5.8) is divergent in the $\epsilon \rightarrow 0$ limit.

It is proposed in [17] that BMSFT entanglement entropy can be found by taking the limit from the CFT counterpart. In the prescription of [17] it is argued that the symmetries are enough to fix the form of the entanglement entropy. Then they use a new contraction of conformal algebra which results in BMS algebra. But the relation between central charges of conformal algebra and BMS algebra differs from (5.4). Since the final algebra is the same as the ultra-relativistic contraction of [12], the author of [17] argued that their results are the entanglement entropy of BMSFT.

If we continue the logic which relates the Cardy-like formula to the limit of inner horizon entropy then we conclude that the entanglement entropy of BMSFT should be related to a formula in the CFT which is transformed to the inner horizon formula by using Rindler transformation [19]. It is not difficult to check that the formula

$S_{\text {inner }}=\frac{c-\bar{c}}{2} \log \frac{R}{\epsilon_{R}}-\frac{c+\bar{c}}{6} \kappa$

results in the leading term of entanglement entropy of zero temperature BMSFT on the plane after taking the ultrarelativistic limit. This formula is nothing but the inner horizon Cardy formula (5.5) if we use Rindler transformation [19]. Therefore we conclude that $S^{0}$ in (4.13) is given by taking limit from a formula which is related to the inner horizon 
of dual spacetime. Similarly, the logarithmic correction in (4.13) is also given by taking limit from a formula which is the logarithmic correction to the inner horizon entropy. It is not difficult to check that taking $\epsilon \rightarrow 0$ limit from

$$
S_{\text {log, inner }}=-\frac{3}{2} \log \left|c^{1 / 3} \frac{\partial S_{0}}{\partial c}\right|-\frac{3}{2} \log \left|\bar{c}^{1 / 3} \frac{\partial S_{0}}{\partial \bar{c}}\right|-\log \epsilon,
$$

results in the logarithmic term of (4.13) up to a constant. The interpretation of (5.10) in the gravity is of importance. As mentioned before $\epsilon$ in the field theory corresponds to $G / \ell$ on the bulk side. For the BTZ black holes, we have

$\beta=\frac{2 \pi \ell}{\left(r_{+}+r_{-}\right)}, \quad \bar{\beta}=\frac{2 \pi \ell}{\left(r_{+}-r_{-}\right)}$.

where $r_{ \pm}$are the radii of inner and outer horizons. Thus, using (5.5), (5.10) and (5.11), we can find the logarithmic correction to the inner horizon entropy of BTZ black hole as

$S_{\text {inner }}^{B T Z}=\frac{\pi r_{-}}{2 G}-\frac{3}{2} \log \frac{r_{+}^{2}-r_{-}^{2}}{\ell^{2}}+$ constant,

where we have substituted central charges as $c=\bar{c}=\frac{3 \ell}{2 G}$. On the other hand, using (5.1), (5.2) and (5.11) we find that

$S_{\text {outer }}^{\text {BTZ }}=\frac{\pi r_{+}}{2 G}-\frac{3}{2} \log \frac{r_{+}^{2}-r_{-}^{2}}{\ell^{2}}-\log \frac{\ell}{G}+$ constant.

It is clear from (5.12) and (5.13) that due to the logarithmic corrections, multiplication of inner and outer horizon entropies depends not only on the angular momentum but also on the mass of BTZ. This corrects the result of [31] which in the leading term the multiplication of inner and outer entropies is mass independent.

\section{Conclusion}

In this paper we introduced a generic formula for the logarithmic corrections of the BMSFT thermal and the entanglement entropies. Our derivation is based on the Rindler method which makes connections between the thermal and the entanglement entropies. The most important application of the present work will reveal itself in the reconstruction of the bulk dynamics beyond the classical regime. This reconstruction has been done recently in the bulk AdS case through the perturbation of the entanglement entropy [26,27]. After generalizing the method of [26] for the flat-space holography, we will be able to derive the quantum corrections to the Einstein gravity without the cosmological constant field equations using the results of the current paper.

Most of the works in the flat-space holography can be performed by taking the flat space-limit from the AdS/CFT calculations. One of the possible roads for finding the corrections of the BMSFT entanglement entropy formula is taking limit from the calculation of [37] which studies the one-loop bulk corrections to the Ryu-Takayanagi formula.

Acknowledgements The authors would like to thank S. M. Hosseini for his useful comments on the manuscript. We are also grateful to Yousef Izadi for his comments on the revised version. We would like to thank the referee for his/her useful comments. This research is supported by research grant No. 600/1476 of Shahid Beheshti University, G.C.

Open Access This article is distributed under the terms of the Creative Commons Attribution 4.0 International License (http://creativecomm ons.org/licenses/by/4.0/), which permits unrestricted use, distribution, and reproduction in any medium, provided you give appropriate credit to the original author(s) and the source, provide a link to the Creative Commons license, and indicate if changes were made. Funded by SCOAP $^{3}$.

\section{References}

1. J.M. Maldacena, The large $\mathrm{N}$ limit of superconformal field theories and supergravity. Int. J. Theor. Phys. 38, 1113 (1999). https://doi. org/10.1023/A:1026654312961. arXiv:hep-th/9711200

2. J.M. Maldacena, The large $\mathrm{N}$ limit of superconformal field theories and supergravity. Adv. Theor. Math. Phys. 2, 231 (1998)

3. H. Bondi, M.G. van der Burg, A.W. Metzner, Gravitational waves in general relativity. 7. Waves from axisymmetric isolated systems. Proc. R. Soc. Lond. A 269, 21 (1962)

4. R.K. Sachs, Gravitational waves in general relativity. 8. Waves in asymptotically flat space-times. Proc. R. Soc. Lond. A 270, 103 (1962)

5. R.K. Sachs, Asymptotic symmetries in gravitational theory. Phys. Rev. 128, 2851 (1962)

6. A. Ashtekar, J. Bicak, B.G. Schmidt, Asymptotic structure of symmetry reduced general relativity. Phys. Rev. D 55, 669 (1997). arXiv:gr-qc/9608042

7. G. Barnich, G. Compere, Classical central extension for asymptotic symmetries at null infinity in three spacetime dimensions. Class. Quantum Gravity 24, F15 (2007). arXiv:gr-qc/0610130

8. G. Barnich, C. Troessaert, Symmetries of asymptotically flat 4 dimensional spacetimes at null infinity revisited. arXiv:0909.2617 [gr-qc]

9. G. Barnich, C. Troessaert, Aspects of the BMS/CFT correspondence. JHEP 1005, 062 (2010). arXiv:1001.1541 [hep-th]

10. A. Bagchi, Correspondence between asymptotically flat spacetimes and nonrelativistic conformal field theories. Phys. Rev. Lett. 105, 171601 (2010)

11. A. Bagchi, The BMS/GCA correspondence. arXiv:1006.3354 [hep-th]

12. A. Bagchi, R. Fareghbal, BMS/GCA Redux: Towards Flatspace Holography from Non-Relativistic Symmetries. arXiv:1203.5795 [hep-th]

13. A. Bagchi, R. Basu, D. Grumiller, M. Riegler, Entanglement entropy in Galilean conformal field theories and flat holography. Phys. Rev. Lett. 114(11), 111602 (2015). https://doi.org/10.1103/ PhysRevLett.114.111602. arXiv:1410.4089 [hep-th]

14. P. Calabrese, J.L. Cardy, Entanglement entropy and quantum field theory. J. Stat. Mech. 0406, P06002 (2004). https://doi.org/10. 1088/1742-5468/2004/06/P06002. arXiv:hep-th/0405152

15. S. Ryu, T. Takayanagi, Holographic derivation of entanglement entropy from AdS/CFT. Phys. Rev. Lett. 96, 181602 (2006). https:// doi.org/10.1103/PhysRevLett.96.181602. arXiv:hep-th/0603001

16. E. Witten, (2+1)-Dimensional gravity as an exactly soluble system. Nucl. Phys. B 311, 46 (1988). https://doi.org/10.1016/ 0550-3213(88)90143-5 
17. S.M. Hosseini, A. Veliz-Osorio, Gravitational anomalies, entanglement entropy, and flat-space holography. Phys. Rev. D 93(4), 046005 (2016). https://doi.org/10.1103/PhysRevD.93. 046005. arXiv:1507.06625 [hep-th]

18. R. Basu, M. Riegler, Wilson lines and holographic entanglement entropy in galilean conformal field theories. Phys. Rev. D 93(4), 045003 (2016). https://doi.org/10.1103/PhysRevD.93. 045003. arXiv:1511.08662 [hep-th]

19. H. Jiang, W. Song, Q. Wen, Entanglement entropy in flat holography. arXiv:1706.07552 [hep-th]

20. H. Casini, M. Huerta, R.C. Myers, Towards a derivation of holographic entanglement entropy. JHEP 1105, 036 (2011). https://doi. org/10.1007/JHEP05(2011)036. arXiv:1102.0440 [hep-th]

21. A. Bagchi, S. Detournay, R. Fareghbal, J. Simn, Holography of 3D flat cosmological horizons. Phys. Rev. Lett. 110(14), 141302 (2013). https://doi.org/10.1103/PhysRevLett.110.141302. arXiv:1208.4372 [hep-th]

22. A. Bagchi, R. Basu, 3D flat holography: entropy and logarithmic corrections. JHEP 1403, 020 (2014). https://doi.org/10.1007/ JHEP03(2014)020. arXiv:1312.5748 [hep-th]

23. S. Carlip, Logarithmic corrections to black hole entropy from the Cardy formula. Class. Quantum Gravity 17, 4175 (2000). https:// doi.org/10.1088/0264-9381/17/20/302. arXiv:gr-qc/0005017

24. A. Sen, Logarithmic corrections to rotating extremal black hole entropy in four and five dimensions. Gen. Rel. Gravity 44, 2012 (1947). https://doi.org/10.1007/s10714-012-1373-0. arXiv:1109.3706 [hep-th]

25. A. Sen, Logarithmic corrections to Schwarzschild and other non-extremal black hole entropy in different dimensions. JHEP 1304, 156 (2013). https://doi.org/10.1007/JHEP04(2013)156. arXiv:1205.0971 [hep-th]

26. T. Faulkner, M. Guica, T. Hartman, R.C. Myers, M. Van Raamsdonk, Gravitation from entanglement in holographic CFTs. JHEP 1403, 051 (2014). https://doi.org/10.1007/JHEP03(2014)051. arXiv:1312.7856 [hep-th]
27. N. Lashkari, M.B. McDermott, M. Van Raamsdonk, Gravitational dynamics from entanglement 'thermodynamics'. JHEP 1404, 195 (2014). https://doi.org/10.1007/JHEP04(2014)195. arXiv:1308.3716 [hep-th]

28. D. Allahbakhshi, M. Alishahiha, A. Naseh, Entanglement thermodynamics. JHEP 1308, 102 (2013). https://doi.org/10.1007/ JHEP08(2013)102. arXiv:1305.2728 [hep-th]

29. R. Fareghbal, A. Naseh, Aspects of flat/CCFT correspondence. Class. Quantum Gravity 32, 135013 (2015). https://doi.org/10. 1088/0264-9381/32/13/135013. arXiv:1408.6932 [hep-th]

30. M. Riegler, Flat space limit of higher-spin Cardy formula. Phys. Rev. D 91(2), 024044 (2015). https://doi.org/10.1103/PhysRevD. 91.024044. arXiv:1408.6931 [hep-th]

31. F. Larsen, A string model of black hole microstates. Phys. Rev. D 56, 1005 (1997). https://doi.org/10.1103/PhysRevD.56.1005. arXiv:hep-th/9702153

32. S. Detournay, Inner mechanics of $3 \mathrm{~d}$ black holes. Phys. Rev. Lett. 109, 031101 (2012). https://doi.org/10.1103/PhysRevLett. 109.031101. arXiv: 1204.6088 [hep-th]

33. G. Barnich, Entropy of three-dimensional asymptotically flat cosmological solutions. JHEP 1210, 095 (2012). https://doi.org/10. 1007/JHEP10(2012)095. arXiv:1208.4371 [hep-th]

34. R. Basu, S. Detournay, M. Riegler, Spectral flow in 3D flat spacetimes. arXiv:1706.07438 [hep-th]

35. F. Loran, M.M. Sheikh-Jabbari, M. Vincon, Beyond logarithmic corrections to Cardy formula. JHEP 1101, 110 (2011). https://doi. org/10.1007/JHEP01(2011)110. arXiv:1010.3561 [hep-th]

36. A. Castro, S. Detournay, N. Iqbal, E. Perlmutter, Holographic entanglement entropy and gravitational anomalies. JHEP 1407, 114 (2014). https://doi.org/10.1007/JHEP07(2014)114. arXiv:1405.2792 [hep-th]

37. T. Barrella, X. Dong, S.A. Hartnoll, V.L. Martin, Holographic entanglement beyond classical gravity. JHEP 1309, 109 (2013). https://doi.org/10.1007/JHEP09(2013)109. arXiv:1306.4682 [hep-th] 